\title{
Study on the relationship between the ternary interaction parameter and the structure of water by the effect of aliphatic alcohols and phenol on the swelling behavior of poly(ethylene-co-vinyl alcohol) membrane in aqueous solution
}

\author{
Liao-Ping Cheng a , Ken-Yu Chang ${ }^{\mathrm{b}}$, Ping-Shan Lai ${ }^{\mathrm{b}}$, Tai-Horng Young ${ }^{\mathrm{b}, *}$ \\ a Department of Chemical Engineering, Tamkang University, Taipei 25137, Taiwan ROC \\ ${ }^{\mathrm{b}}$ Institute of Biomedical Engineering, College of Medicine and College of Engineering, National Taiwan University, No.1, Sec. 1, \\ Jen-Ai Road, Taipei 10016, Taiwan ROC
}

Received 3 December 2003; received in revised form 6 June 2004; accepted 21 June 2004

Available online 26 August 2004

\begin{abstract}
The effect of very low concentrations of ethanol, 2-propanol and phenol on the swelling degree of poly(ethylene-covinyl alcohol) (EVAL) in water was investigated. The effect of phenol on the swelling degree of EVAL was remarkably large compared to that of ethanol and that of 2-propanol. Theoretical analysis on the basis of Flory-Huggins theory using three binary interaction parameters could appropriately predict the EVAL swelling degree in ethanol/water and 2-propanol/water mixtures. However, the theoretical swelling degree of EVAL in phenol/water mixtures needed a ternary interaction parameter $\left(\chi_{T}\right)$ to match with experimental data points. An optimum value of $\chi_{T}$ for the water-phenolEVAL system was found to be -3.3 . The relationship between the ternary interaction parameter and the structure of water from observations of the effect of phenol on the EVAL swelling was discussed. Based on the analysis of low-frequency Raman spectroscopy reported by Suzuki et al. [J. Chem. Phys. 107 (1997) 5890], the contribution of $\chi_{T}$ to the EVAL swelling was attributed to the increase of the entropy in bulk water due to the effect of phenol on the disruption of the tetrahedral hydrogen-bonded networks of water molecules. This, in turn, induced an increase of water absorption in EVAL.

(C) 2004 Elsevier Ltd. All rights reserved.
\end{abstract}

Keywords: EVAL swelling; Ternary interaction parameter; Water structure

\footnotetext{
* Corresponding author. Tel.: +886 223123 456x1455; fax: +886223940049.

E-mail address: thyoung@ha.mc.ntu.edu.tw (T.-H. Young).
}

\section{Introduction}

The extensive development of industrial separation process using membranes has focused the attention on different membrane properties. For example, the equilibrium swelling degree of a membrane is an important property, which influences the membrane's permeability, 
selectivity and mechanical properties. Furthermore, a membrane swollen in water is of considerable importance for the application of drug delivery and pervaporation. Therefore, a great deal of effort has been paid to study the swelling behavior of polymers [1-5]. Any compound that is present in water, however, also can influence the swelling behavior of polymers if it binds to the polymer or changes the water property. Therefore, it has become necessary to perform more fundamental investigation on the mechanisms at the microscopic level governing the membrane swelling behavior.

In a previous study [6], the swelling behavior of poly(ethylene-co-vinyl alcohol) (EVAL), polyurethane and poly(ethylene-co-vinyl acetate) in ethanol/water mixtures was investigated. It was found that the hydrophobic interaction between polymer and water is a major factor to influence the polymer swelling due to changes in the structuring of water around hydrophobic polymers. Therefore, it is important to understand the water structure around a polymer membrane because the water structure will influence the membrane swelling behavior to a great degree. In this study, the effect of ethanol, 2-propanol and phenol on the structure of water from observations of their effect on the swelling behavior of EVAL was investigated. All substances studied here, added in tiny amounts, increased the swelling behavior of EVAL. The effect of phenol on the EVAL swelling was much stronger than that of ethanol and that of 2-propanol. In order to get a better understanding of membrane-water interactions in the presence of other compounds, the difference in swelling behavior of EVAL was studied by the theoretical analysis of competitive interactions on the basis of a suitable thermodynamic model. Most attempts to analyze the phase behavior of ternary systems are based on a consideration of the binary interaction parameter embodied in the Flory-Huggins theory [7]. However, it was found that a ternary interaction parameter $\left(\chi_{T}\right)[8,9]$ was required for an accurate description of the effect of phenol on the EVAL swelling behavior, suggesting that binary interaction parameters are not the controlling parameter in this system and that there exists an additional interaction among phenol, water and EVAL. It is well known that a ternary interaction parameter is required for an accurate description of the preferential sorption of polymers in mixed solvents [10,11]. However, the mechanism for the existence of the ternary interaction parameter remains to be interpreted. Therefore, through comparing theoretical predictions and experimentally obtained data, the physical meaning of $\chi_{T}$ and the mechanism for phenol to significantly influence the swelling degree of EVAL in water is discussed in this work.

\section{Materials and methods}

EVAL was obtained from Kuraray Co. Ltd., Japan (EP-E105A) having an average ethylene content of 44 $\mathrm{mol} \%$ and an average molecular weight $M_{\eta}=56,000 \mathrm{~g} /$ mol (intrinsic viscosity $=0.87 \mathrm{dl} / \mathrm{g}$ ) [12]. The EVAL polymer used for swelling measurements in the form of a membrane with a dense structure was prepared by solvent evaporation in a vacuum oven [13]. A piece of membrane, of a known weight, was immersed into a flask containing water and very low concentrations of ethanol, 2-propanol or phenol at $25{ }^{\circ} \mathrm{C}$ for several weeks. The changes in the weights of the membranes were measured by carefully wiping the superfluous liquid with tissue paper. The swelling equilibrium was established until no further weight increase was observed. The swelling degree was expressed as a relative weight increase (gram of liquid/gram of dry membrane).

\subsection{Simulation theory}

Besides experimental observation, the effect of ethanol, 2-propanol and phenol on the swelling degree of EVAL in water was predicted theoretically. In this study, the Flory-Huggins theory [7] was used to describe the Gibbs free energy of mixing $\left(\Delta G_{\mathrm{m}}\right)$ for a ternary system, in which a ternary interaction parameter, $\chi_{T}$, was included:

$$
\begin{aligned}
\Delta G_{\mathrm{m}}= & R T\left(n_{1} \ln \phi_{1}+n_{2} \ln \phi_{2}+n_{3} \ln \phi_{3}+\chi_{12} n_{1} \phi_{2}\right. \\
& \left.+\chi_{13} n_{1} \phi_{3}+\chi_{23} n_{2} \phi_{3}+\chi_{T} n_{1} \phi_{2} \phi_{3}\right)
\end{aligned}
$$

where $n_{i}$ is the mole of the component $i, \phi_{i}$ is its volume fraction, $\chi_{i j}$ is the binary interaction parameter between the components $i$ and $j$. For the present ternary system, the subscript $i=1,2$, and 3 denotes water, added substance, and EVAL, respectively. Differentiating Eq. (1) with respect to $n_{1}$ and $n_{2}$, the chemical potential, $\Delta \mu_{i}\left(\mu_{i} \mu_{i}^{0}\right)$ in the membrane phase is given:

$$
\begin{aligned}
\frac{\Delta \mu_{1}}{R T}= & \ln \phi_{1}+1-\phi_{1}-\frac{V_{1}}{V_{2}} \phi_{2}-\frac{V_{1}}{V_{3}} \phi_{3} \\
& +\left(\phi_{2} \chi_{12}+\phi_{3} \chi_{13}\right)\left(\phi_{2}+\phi_{3}\right)-\frac{V_{1}}{V_{2}} \phi_{2} \phi_{3} \chi_{23} \\
& -h_{1} h_{2} \phi_{2} \frac{\mathrm{d} \chi_{12}}{\mathrm{~d} h_{2}}-\phi_{1} \phi_{2} \phi_{3} \frac{\partial \chi_{13}}{\partial \phi_{2}}-\phi_{1} \phi_{3}^{2} \frac{\partial \chi_{13}}{\partial \phi_{2}} \\
& -\phi_{1} \phi_{3}^{2} \frac{\partial \chi_{13}}{\partial \phi_{3}}-\frac{V_{1}}{V_{2}} \phi_{2}^{2} \phi_{3} \frac{\partial \chi_{23}}{\partial \phi_{2}}-\frac{V_{1}}{V_{2}} \phi_{2} \phi_{3}^{2} \frac{\partial \chi_{23}}{\partial \phi_{3}} \\
& -\phi_{1} \phi_{2}^{2} \phi_{3} \frac{\partial \chi_{T}}{\partial \phi_{2}}-\phi_{1} \phi_{2} \phi_{3}^{2} \frac{\partial \chi_{T}}{\partial \phi_{3}}-\chi_{T} \phi_{2} \phi_{3}\left(1-2 \phi_{1}\right)
\end{aligned}
$$




$$
\begin{aligned}
\frac{\Delta \mu_{2}}{R T}= & \ln \phi_{2}+1-\phi_{2}-\frac{V_{2}}{V_{1}} \phi_{1}-\frac{V_{2}}{V_{3}} \phi_{3} \\
& +\left(\frac{V_{2}}{V_{1}} \phi_{1} \chi_{12}+\phi_{3} \chi_{23}\right)\left(\phi_{1}+\phi_{3}\right) \\
& -\frac{V_{2}}{V_{1}} \phi_{1} \phi_{3} \chi_{13}+\frac{V_{2}}{V_{1}} h_{1} h_{2} \phi_{1} \frac{\mathrm{d} \chi_{12}}{\mathrm{~d} h_{2}} \\
& +\frac{V_{2}}{V_{1}} \phi_{1} \phi_{3}\left(\phi_{1}+\phi_{3}\right) \frac{\partial \chi_{13}}{\partial \phi_{2}}-\frac{V_{2}}{V_{1}} \phi_{1} \phi_{3}^{2} \frac{\partial \chi_{13}}{\partial \phi_{3}} \\
& +\phi_{2} \phi_{3}\left(\phi_{1}+\phi_{3}\right) \frac{\partial \chi_{23}}{\partial \phi_{2}}-\phi_{2} \phi_{3}^{2} \frac{\partial \chi_{23}}{\partial \phi_{3}} \\
& +\frac{V_{2}}{V_{1}} \phi_{1} \phi_{2} \phi_{3}\left(\phi_{1}+\phi_{3}\right) \frac{\partial \chi_{T}}{\partial \phi_{2}}-\frac{V_{2}}{V_{1}} \phi_{1} \phi_{2} \phi_{3}^{2} \frac{\partial \chi_{T}}{\partial \phi_{3}} \\
& +\frac{V_{2}}{V_{1}} \chi_{T} \phi_{1} \phi_{3}\left(1-2 \phi_{2}\right)
\end{aligned}
$$

where $\mu_{i}^{0}$ is the chemical potential of component $i$ at standard state, $V_{i}$ is the molar volume, $h_{1}=\phi_{1} /$ $\left(\phi_{1}+\phi_{2}\right)$ and $h_{2}=\phi_{2} /\left(\phi_{1}+\phi_{2}\right)$. Likewise, the chemical potentials, $\Delta \mu_{1}$ and $\Delta \mu_{2}$, in the liquid mixture phase can be obtained according to Eqs. (2) and (3) by assuming $\phi_{3}=0$.

At equilibrium between the liquid mixture phase and the membrane phase ( 1 and $\mathrm{m}$ ) at a specified temperature and pressure, the chemical potentials of each liquid component in these two phases are equal, i.e.,

$\mu_{1}^{1}=\mu_{1}^{\mathrm{m}}$

$\mu_{2}^{1}=\mu_{2}^{\mathrm{m}}$

where $\mu_{i}^{1}$ and $\mu_{i}^{\mathrm{m}}$ are the chemical potentials of component $i$ in the liquid mixture phase and the membrane phase, respectively. Since volume fractions of all components in the membrane phase $\left(\phi_{i}^{\mathrm{m}}\right)$ add up to 1 , we have:

$\phi_{1}^{\mathrm{m}}+\phi_{2}^{\mathrm{m}}+\phi_{3}^{\mathrm{m}}=1$

Eqs. (2)-(6) describe the compositions of the membrane phase at equilibrium. Given interaction parameters and related physical properties, these equations can be used to compute the theoretical membrane swelling degree $\left(\left(\rho_{1} \phi_{1}^{\mathrm{m}}+\rho_{2} \phi_{2}^{\mathrm{m}}\right) / \rho_{3} \phi_{3}^{\mathrm{m}}\right)$, where $\rho_{i}$ is the density of component $i$.

\subsection{Interaction parameters}

In this study, all $\chi_{12}$ parameters between water and added substances at $25{ }^{\circ} \mathrm{C}$ were calculated from the excess Gibbs energy data using the group contribution method of UNIFAC [14]. Assuming that $\chi_{12}$ has the functional form suggested by Koningsveld and Kleintjens [15], parameters $a, b$, and $c$ in Eq. (7) could be found by least square regression.

$\chi_{12}=a-\frac{b}{1-c \phi_{2}}$
For a ternary system, following Yilmaz and McHugh [16], $\chi_{12}$ was assumed to be a function only of $\phi_{2}$ / $\left(\phi_{1}+\phi_{2}\right)$. Thus, $\chi_{12}$ was obtained by replacing $\phi_{2}$ with $H_{2}=\phi_{2} /\left(\phi_{1}+\phi_{2}\right)$ in Eq. (7).

Binary interaction parameters between pure liquid and polymer, $\chi_{13}$ and $\chi_{23}$, were determined using data from equilibrium swelling experiments of the pure components in the EVAL membrane [17]. The following equation was employed:

$\ln \phi_{i}+\left(1-\frac{1}{x_{n}}\right) \phi_{3}+\chi_{i 3} \phi_{3}^{2}=0, i=1$ or 2

where $x_{n}$ is the degree of polymerization of the polymer. Since phenol is solid at $25^{\circ} \mathrm{C}$, the binary interaction parameter between phenol and EVAL was obtained by the solubility parameter method [18]. The following equation was employed:

$\chi_{23}=\frac{V_{2}\left(\delta_{2}-\delta_{3}\right)^{2}}{R T}$

where $\delta$ is the solubility parameter. The solubility parameters of phenol and EVAL are 24.1 and 27.6 $\left(\mathrm{MPa}^{0.5}\right)$, respectively $[19,20]$.

Experimental data for the ternary interaction parameter, $\chi_{T}$ is not available in literature. In this study, we simply treated $\chi_{T}$ as an empirical correction parameter. A fitting procedure was performed by varying $\chi_{T}$ to see if the theoretical membrane swelling fits the experimental data.

\section{Results}

The EVAL swelling for the addition of the various substances in aqueous solution at $25{ }^{\circ} \mathrm{C}$ were plotted

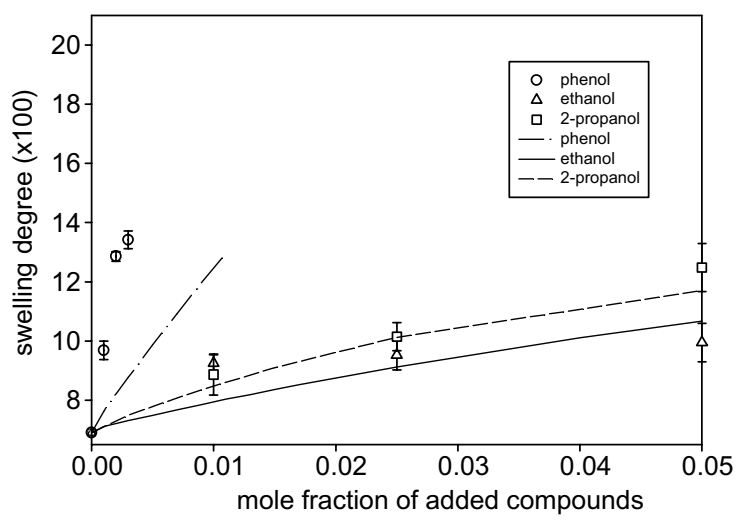

Fig. 1. Experimental EVAL swelling data and theoretical curves according to the Flory-Huggins theory with $\chi_{T}=0$ for the addition of ethanol, 2-propanol and phenol in aqueous solution at $25^{\circ} \mathrm{C}$. 
as a function of molar fraction in Fig. 1. All data were shown as the mean \pm standard deviation from triplicate independent experiments. A previous paper [6] has shown that the shape of the swelling curves is quite different for different polymers in water/ethanol mixtures. In this work, all added substances studied here increased the EVAL swelling and the swelling degree increased with increasing the concentration of added substances. Although the differences in swelling degree of EVAL in ethanol and 2-propanol aqueous solutions are not very significant, it is obvious that the effect of phenol on the EVAL swelling is stronger than that of ethanol and that of 2-propanol.

As mentioned in the simulation theory, the swelling behavior of a polymer can be derived from the FloryHuggins theory with a number of parameters. Therefore, theoretical EVAL swelling curves determined by solving Eqs. (2)-(6) with $\chi_{T}=0$, together with the experimental data points for comparison, are given in Fig. 1. The physical constants employed in swelling computations are given in Table 1 . The binary interaction parameters, $\chi_{i j}$, were found by using Eqs. (7)-(9) and the results are given in Table 2. Fig. 1 shows the results of theoretical swelling curve with $\chi_{T}=0$ appear to be in line with experimental findings for EVAL in water/ethanol and water/2-propanol mixtures. In the water/phenol mixture, although both the experimental and the theoretical swelling values with $\chi_{T}=0$ show a similar trend, the theoretical values are notably smaller than the experimental data. This indicates that only binary interaction parameters approach to the EVAL swelling in the water/phe-

Table 1

Physical properties of EVAL, water, ethanol, 2-propanol and phenol

\begin{tabular}{lcll}
\hline Component & $\begin{array}{l}\text { Molecular } \\
\text { weight }\end{array}$ & $\begin{array}{l}\text { Density } \\
\left(\mathrm{g} / \mathrm{cm}^{3}\right)\end{array}$ & $\begin{array}{l}\text { Molar } \\
\text { volume }\left(\mathrm{cm}^{3} / \mathrm{mol}\right)\end{array}$ \\
\hline EVAL & 56,000 & 1.14 & 47,863 \\
Water & 18 & 1 & 18 \\
Ethanol & 44 & 0.78 & 58.23 \\
2-Propanol & 60 & 0.784 & 76.53 \\
Phenol & 94 & 1.06 & 84.85 \\
\hline
\end{tabular}

Table 2

Summary of binary interaction parameters at $25^{\circ} \mathrm{C}$

\begin{tabular}{ll}
\hline & $\chi_{i j}$ \\
\hline Water-EVAL & 1.956 \\
Water-ethanol & $2.7521-1.7971 /\left(1+0.1346 * \mathrm{H}_{2}\right)$ \\
Water-2-propanol & $0.4317+0.6988 /\left(1.0-0.4386 * \mathrm{H}_{2}\right)$ \\
Water-phenol & $1.3889-0.4308 /\left(1+2.4994 * \mathrm{H}_{2}\right)$ \\
Ethanol-EVAL & 1.51 \\
2-propanol-EVAL & 2.1 \\
Phenol-EVAL & 0.438 \\
\hline
\end{tabular}

$H_{2}=\phi_{2} /\left(\phi_{1}+\phi_{2}\right)$.

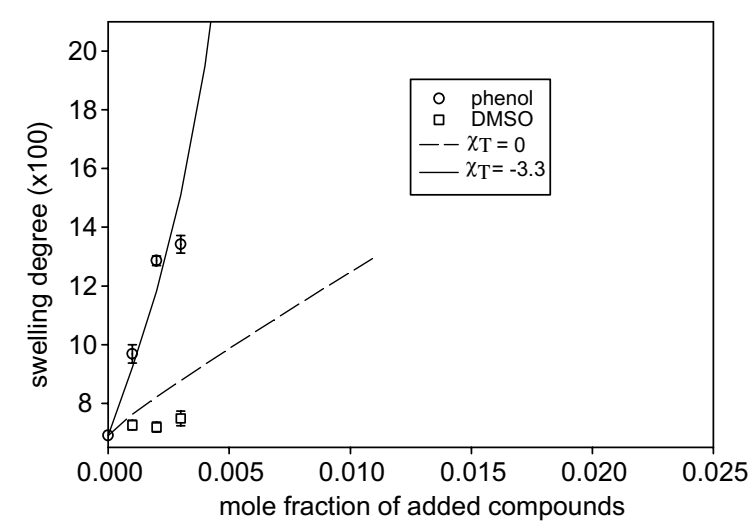

Fig. 2. Experimental EVAL swelling data and theoretical curves according to the Flory-Huggins theory with $\chi_{T}=0$ and -3.3 for the addition of phenol in aqueous solution at 25 ${ }^{\circ} \mathrm{C}$. For comparison, experimental EVAL swelling data for the addition of DMSO in aqueous solution at $25^{\circ} \mathrm{C}$ are shown.

nol mixture is inadequate. To improve the agreement between the experimental and theoretical values, we postulated the existence of a ternary interaction parameter, $\chi_{T}$ to modify the theoretical EVAL swelling in the water/ phenol mixture. The main difficulty in applying the concentration-dependent $\chi_{T}$ parameter to a ternary system is to measure the $\chi_{T}$ parameter directly. For simplicity, due to very low concentrations of added phenol molecules, the theoretical swelling behavior of EVAL in phenol aqueous solution was calculated by selecting $\chi_{T}$ being a constant to examine whether or not $\chi_{T}$ is a significant factor. Fig. 2 shows the theoretical swelling curve of EVAL in phenol aqueous solution fits well to experimental data points for $\chi_{T}=-3.3$, suggesting that a reliable curve fitting has been obtained. Therefore, it is appropriate to introduce a ternary interaction parameter when binary parameters provide an inadequate description of swelling behavior.

\section{Discussion}

The original Flory-Huggins relation generalized for three-component systems is a special case of Eq. (1) where $\chi_{T}$ is equal to zero. For the theoretical analysis of our experimental data, we tried first to employ the simple relation for $\Delta G_{\mathrm{m}}$, i.e., $\chi_{T}=0$. However, the EVAL swelling behavior in phenol aqueous solution cannot be predicted by the binary interaction parameters only, so we postulated the existence of a ternary interaction parameter to rationalize this behavior. Similarly, the ternary interaction parameter is required for an accurate description of the preferential sorption of polymers in mixed solvents $[10,11]$. Basically, the ternary interaction parameters cannot be evaluated from experimental data of independent binary systems. Thus, 
the ternary interaction parameter can be considered as an adjustable one to experimental data and encompasses all deviations of the real system from the idealized system for which only the binary terms are considered $[21,22]$. Munk et al. calculated the ternary interaction parameters in the range between -0.2 and 0.8 for the preferential sorption of polymers in different mixed solvents $[21,22]$.

In this study, an appropriate agreement for phenol to influence the swelling degree of EVAL in water was obtained by using $\chi_{T}=-3.3$. Similar to the binary interaction parameter, the parameter $\chi_{T}$ with a negative value has a positive effect on the polymer swelling [6]. However, what is the mechanism for phenol to influence the swelling degree of EVAL in water to a large degree? The physical meaning of such a highly negative $\chi_{T}$ remains to be interpreted. It is known that the Flory-Huggins interaction parameter $\chi_{i j}$ is, by its original definition, a measure of the interaction energy between each polymer segment and its neighbors [7]. Nonetheless, the Flory-Huggins interaction parameter $\chi_{i j}$ is defined better as a measure of the excess Gibbs energy of a binary system [7]. Thus, in analyzing the meaning of $\chi_{T}$ it is necessary to take into account both of the contribution of enthalpy and entropy for the formation of the ternary complex (1-2-3).

At first, the contribution of enthalpy is considered. For a negative $\chi_{T}$ parameter, it is assumed that the formation of $(1-2-3)$ contacts is energetically favorable. Therefore, one may assume a coupling effect: phenol molecules show a favorite orientation wherein the hydrophobic parts point in the direction of the polymer and the hydrophilic parts point in the direction of the water molecules. Hence, when phenol permeates into EVAL, water is incorporated with phenol to permeate into EVAL. In this study, only very low concentration of phenol was added to water and the OH-group of phenol only can be hydrogen-bonded to limited water molecules [23], thus, it is not reasonable that phenol in tiny amounts can create a high EVAL swelling. Consequently, the coupling effect is not appropriate to explain the result of a very negative $\chi_{T}$ value.

On the other hand, one may assume that a higher amount of water permeates into EVAL after phenol swelling EVAL. Recent measurements in the crosslinked poly( $N$-isopropylacrylamide) gel swollen in phenol-water mixtures showed that when the elastic term is taken into account, the presence of phenol changes the value of the third order interaction parameter [24]. In the present system, it is expected that polyethylene sequences in the EVAL cluster into aggregates, which act as cross-links, so the swelling degree of the membranes being limited by the effective elastic modulus of the network. In the presence of phenol, some of the polyethylene aggregates partly dissolve, thereby reducing the elastic modulus and increasing the swelling degree. Such a mechanism was checked by using DMSO to replace phenol to repeat the swelling experiment; see Fig. 2. Although DMSO is a good solvent for EVAL [12], the concentration dependence of the EVAL swelling from DMSO solutions was much smaller than that from phenol solutions. In contrast, the DMSO concentration dependence of the EVAL swelling was similar to the ethanol and 2-propanol. Therefore, it is not reasonable that phenol in tiny amounts can, but DMSO cannot, partly dissolve the EVAL networks to create a high EVAL swelling.

Again, one may assume that phenol is a weak acid, so the adsorbed phenol molecules can split of protons and then negatively charge the polymer chain, which leads to the membrane swelling due to electric charge repulsion. Such an ionization effect was checked by measuring the EVAL swelling degree in water at various $\mathrm{pH}$. As shown in Fig. 3, it can be seen that the membrane swelling does not increase until the $\mathrm{pH}$ decreases to 4.0 , which is much stronger than a weak acid. Therefore, the property of weak aromatic acid is also not inappropriate to explain the result of a very negative $\chi_{T}$ value.

The low EVAL swelling in water, shown in Fig. 1, is believed to arise from the hydrophobic property of EVAL. It is known that the water molecules in the vicinity of the hydrophobic polymer form clathrate structures according to the flickering-cluster model [25]. Such hydrophobic hydration clusters due to the formation of hydrogen bonds among water molecules are more stable and ordered than the water clusters in bulk water, which can minimize the net free energy of the system. This implies that the low equilibrium absorption of water in the hydrophobic polymer can be attributed to the ordered structure of water molecules around the EVAL. Thus, in order to understand the EVAL swelling behavior in the phenol aqueous solution, we must pay attention not only the interaction between EVAL and

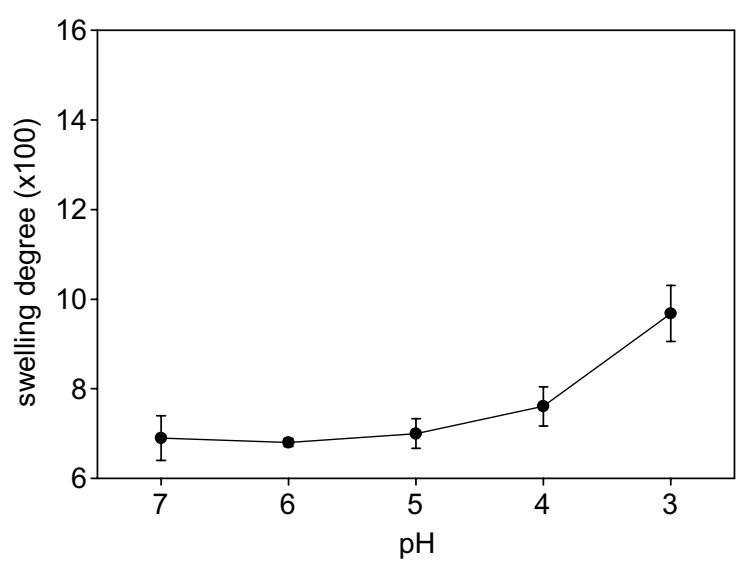

Fig. 3. Experimental EVAL swelling data in water at various $\mathrm{pH}$. 
water in the presence of phenol but also to the effect of phenol on the change in the entropy of the water. In the following, we would like to propose one possible mechanism for $\chi_{T}<0$ caused by the change in entropy of water. If there exists an additional interaction between water and a "water-structure making" substance, the water cluster would be formed firmly and with a larger size, leading to a smaller absorption. In contrast, if the water cluster is destroyed in the presence of a "waterstructure breaking" substance, the water cluster structure would be disrupted to form a new water cluster with a smaller number of water molecules. Therefore, the polymer would adsorb water more easily, leading to a higher swelling degree. Consequently, whether or not the ordered and organized water cluster would be destroyed or strengthened by the addition of other substances to the water is crucial in gaining detailed knowledge of the entropy of water and the swelling behavior of a hydrophobic polymer.

The interpretation for the contribution of entropy for the origin of the negative $\chi_{T}$ parameter is based on the analysis of low-frequency Raman spectroscopy performed by Suzuki et al. [26]. Raman spectroscopy is a powerful method for studying the dynamical structure of the hydrogen-bonded water molecules. In the low-frequency range, Raman spectra contain two vibrational modes of the restricted translations of hydrogen-bonded network of water molecules around 60 and $190 \mathrm{~cm}^{-1}$, corresponding to the bending motion of the $\mathrm{O}-\mathrm{O}-\mathrm{O}$ units containing no less than three water molecules and the stretching of the $\mathrm{O}-\mathrm{H}-\mathrm{O}$ units in the hydrogenbonded network with at least five water molecules, respectively [26]. Therefore, the effect of phenol on the formation of ordered structures of water molecules could be investigated by low-frequency Raman spectroscopy around these two vibrational modes. Suzuki et al. [26] found that presence of the phenol and alcohol molecules at very low concentrations has a considerable effect on the structure of hydrogen-bonded networks in liquid water. They found that the intensity of the bending mode around $60 \mathrm{~cm}^{-1}$ is increased but the intensity of the stretching mode around $190 \mathrm{~cm}^{-1}$ is decreased by the addition of phenol or alcohols into water. Therefore, the structure of the hydrogen-bonded network surround an individual alcohol molecule differs from the structure of bulk water. Instead, it is formed from hydrogenbonded units consisting of three water molecules. This suggests that the tetrahedral structure of water molecules is disrupted and the entropy of water molecules becomes larger. Furthermore, Suzuki et al. [26] found that the change in the entropy of bulk water induced by adding phenol molecules is much larger than the change induced by adding aliphatic alcohol molecules. Such a large effect of phenol on the structure of water clusters is in good agreement with our result that the effect of phenol molecules on the EVAL swelling is much stronger than that of ethanol and that of 2-propanol at the same concentration. From the decrease of tetrahedral structure and the increase of entropy in bulk water, we postulate that phenol has a greater tendency than ethanol and 2-propanol to destroy the tetrahedral structure of water and to form new hydrogen bonded cluster with less water molecules. Therefore, the effect of phenol on increasing the entropy of the bulk water for $\chi_{t}<0$ is proposed.

\section{Conclusion}

Although the $\chi_{T}$ value is only a fitted parameter, the result indicates that $\chi_{T}$ cannot be neglected and might even predict the effect of the added substance on the structure of nearby water clusters to dominate the membrane swelling behavior. Here, we again mention that $\chi_{T}<0$ can be caused also by the change in the enthalpy of the system. Nevertheless, we would like to stress that hydration cluster shells, through hydrogen bonding, form in the vicinity of the added substances contribute to the change of the polymer swelling directly. Of course, it is difficult to understand the concrete structure of the cluster shells around phenols and the mechanism that produces the drastic change in the water structure by the addition of small concentrations of phenol molecules, but the change in the EVAL swelling resulting from the added substance appears to be a good indicator of the effect of the substance on the structure of nearby water clusters in very dilute aqueous solutions.

\section{Acknowledgment}

The authors thank the National Science Council of the Republic of China for their financial support.

\section{References}

[1] Peppas NA, Reinhart CT. Solute diffusion in swollen membranes, I. A new theory. J Membrane Sci 1983;15:275-87.

[2] Mulder MHV, Franken T, Smolders CA. Preferential sorption versus preferential permeability in pervaporation. J Membrane Sci 1985;22:155-73.

[3] Chen SX, Lostritto RT. Diffusion of benzocaine in poly(ethylene-vinyl acetate) membranes: effects of vehicle ethanol concentration and membrane vinyl acetate. $\mathbf{J}$ Controlled Release 1996;38:185-91.

[4] Sen M, Guven O. Prediction of swelling behaviour of hydrogels containing diprotic acid moieties. Polymer 1998;39:1165-72.

[5] Kayaman N, Okay O, Baysal BM. Swelling of polyacrylamide gels in polyacrylamide solutions. J Polym Sci Polym Phys 1998;36:1313-20.

[6] Chuang WY, Young TH, Wang DM, Luo RL, Sun YM. Swelling behavior of hydrophobic polymers in water/ ethanol mixtures. Polymer 2000;41:8339-47. 
[7] Flory P. Principles of polymer chemistry. Ithaca, New York: Cornell University Press; 1953.

[8] Pouchly J, Zivny A. Correlation of data on preferential sorption using the modified Flory-Huggins equation. Makromol Chem 1982;183:3019-40.

[9] Young TH, Cheng LP, Hsieh CC, Chen LW. Phase behavior of EVAL polymers in water-2-propanol cosolvent. Macromolecules 1998;31:1229-35.

[10] Horta A, Gargallo L, Radic D. Preferential adsorption determined by specific interactions. Poly(dialkyl itaconates)/1,4-dioxane/methanol. Macromolecules 1990;23: $5320-5325$.

[11] Campos A, Gavara R, Tejero R, Gomez C, Celda BJ. A procedure for predicting sorption equilibrium in ternary polymer systems from Flory-Huggins binary interaction parameters and the inversion point of preferential solvation. J Polym Sci Polym Phys 1989;27:1599-610.

[12] Young TH, Lai JY, Yu WM, Cheng LP. Equilibrium phase behavior of the membrane forming water-DMSOEVAL copolymer system. J Membrane Sci 1997;128:55-65.

[13] Young TH, Lin DT, Chen LY, Huang YH, Chiu WY. Membranes with a particulate morphology prepared by a dry-wet casting process. Polymer 1999;40:5257-64.

[14] Frednslund A, Gmehling J, Michelsen L, Rasmussen P, Prausnitz JM. Computerized design of multicomponent distillation columns using the UNIFAC group contribution method for calculation of activity coefficients. Ind Eng Chem Process Des Dev 1977;16:450-62.

[15] Koningsveld R, Kleintjens LA. Liquid-liquid phase separation in multicomponent polymer systems. X. Concentration dependence of the pair-interaction parameter in the system cyclohexane-polystyrene. Macromolecules 1971;4: 637-641.
[16] Yilmaz L, McHugh AJ. Analysis of nonsolvent-solventpolymer phase diagrams and their relevance to membrane formation modeling. J Appl Polym Sci 1986;31: 997-1018.

[17] Bonner D. Vapour-liquid equilibria in concentrated polymer solutions. J Macromol Sci Rev Macromol Chem 1975;13:263-319.

[18] Hildebrand J, Scott R. Solubility of nonelectrolytes. 3rd ed. New York, NY: Reinhold; 1949.

[19] Barton AFM. CRC handbook of solubility parameters and other cohesion parameters. CRC Press; 1988.

[20] Chen LW, Young TH. Effect of nonsolvents on the mechanism of wet-casting membrane formation from EVAL copolymers. J Membrane Sci 1991;59:15-26.

[21] Chu SG, Munk P. Thermodynamic properties of polystyrene in mixed solvents studied by sedimentation equilibrium. Macromolecules 1978;11:879-88.

[22] Aminabhavi TM, Munk P. Preferential adsorption onto polystyrene in mixed solvent systems. Macromolecules 1979;12:607-13.

[23] Gerhards M, Kleinermanns K. Structure and vibrations of phenol $\left(\mathrm{H}_{2} \mathrm{O}\right)_{2}$. J Chem Phys 1995;103:7392-400.

[24] Laszlo K, Kosik K, Rochas C, Geissler E. Phase transition in poly $(N$-isopropylacrylamide) hydrogels induced by phenols. Macromolecules 2003;36:7771-6.

[25] Kesting RE. Synthetic polymer membranes. 2nd ed. New York: John Wiley \& Sons; 1985.

[26] Suzuki Y, Suzuki N, Takasu Y, Nishio I. A study on the structure of water in an aqueous solution by the solvent effect on a volume phase transition of $N$-isopropylacrylamide gel and low-frequency Raman spectroscopy. J Chem Phys 1997;107:5890-7. 